which was defined as the most recent IORRA survey before planning pregnancy. The mixed-effect model for repeated measures was used to analyze group difference.

Results: There were 40 patients in the PP group (average 32.2 years, disease duration 5.7 years, DAS28-CRP 1.7, J-HAQ 0.26), and 120 patients in the control group (average 32.4 years, disease duration 5.9 years, DAS28CRP 1.7, J-HAQ 0.21). The proportion of user and dosage of MTX and glucocorticoid (GC) and bDMARDs user at baseline were comparable between the groups (MTX: PP 87.5\% [9.8 mg/week], control 85.0\% [8.8 mg/week]; GC: PP $32.5 \%$ [3.6 mg/day], control 27.5\% [4.4 mg/day]; bDMARDs: PP 40.0\%, control $27.5 \%$ ). DAS28-CRP at year 3 of the PP group elevated and was higher than the control group (PP 2.3, control 1.7, p<0.01), while J-HAQ was stable over the observation period and did not differ significantly at year 3 (PP 0.21 , control $0.22, p=0.92$ ). At year 3 , the proportion of patients taking MTX was lower and taking GC was higher in the PP group than those in the control group (MTX: PP $36.7 \%$, control 76.7\%, p<0.01; GC: PP $70.0 \%$, control $25.6 \%, p<0.01)$. The proportion of patients taking bDMARDs was not different in both groups (PP $36.7 \%$, control $32.6 \%, p=0.68)$.

Conclusion: Physical function in pregnancy planning patients with RA did not deteriorate as well as the control patients in clinical settings.

References:

[1] Arthritis Rheum. 2011;63:1517-1521.

[2] Ann Rheum Dis. 2015;10:1836-1841.

[3] J Rheumatol. 2015;42:1376-1382.

[4] J Rheumatol. 2019;46:245-250.

[5] Arthritis Care Res. 2017;69:1297-1303.

Disclosure of Interests: Moeko Ochiai: None declared, Eiichi Tanaka Consultant of: ET has received lecture fees or consulting fees from Abbvie, Asahi Kasei pharma co., Bristol Myers Squibb, Chugai Pharmaceutical, Daiichi Sankyo Co., Eisai Pharmaceutical, Janssen Pharmaceutical K.K. Nippon Kayaku, Pfizer, Takeda Pharmaceutical, Taisho Toyama Pharmaceutical Co., and UCB Pharma., Speakers bureau: ET has received lecture fees or consulting fees from Abbvie, Asahi Kasei pharma co., Bristol Myers Squibb, Chugai Pharmaceutical, Daiichi Sankyo Co., Eisai Pharmaceutical, Janssen Pharmaceutical K.K., Nippon Kayaku, Pfizer, Takeda Pharmaceutical, Taisho Toyama Pharmaceutical Co., and UCB Pharma., Eisuke Inoue Speakers bureau: El has received speaker fee from Bristol-Meyers, Pfizer, Merck serono., Mai Abe: None declared, Eri Sugano: None declared, Naohiro Sugitani: None declared, Kumiko Saka: None declared, higuchi yoko: None declared, Rei Yamaguchi: None declared, Naoki Sugimoto: None declared, Ikari Katsunori Speakers bureau: KI has received speaker's fee from Asahi Kasei Pharma Corp., Astellas Pharma Inc., AbbVie Japan GK, Ayumi Pharmaceutical Co., Bristol Myers Squibb Co., Ltd., Chugai Pharmaceutical Co., Ltd., Eis, ai Co., Ltd., Eli Lilly Japan K.K., Janssen Pharmaceutical K.K., Kaken Pharmaceutical Co. Ltd., Mitsubishi Tanabe Pharma Corp.Pfizer Japan Inc., Takeda Pharmaceutical Co. Ltd., Teijin Pharma Ltd and UCB Japan Co. Ltd., Ayako Nakajima Grant/research support from: AN has received research grants from Chugai Pharmaceutical Co., Ltd., Mitsubishi Tanabe Pharma Co., Pfizer Japan Inc., Consultant of: AN has consultant fee from Nippon Kayaku Co. Ltd., Speakers bureau: AN has received speaker's fee from AbbVie Japan GK, Actelion Pharmaceuticals Japan LTD., Asahi Kasei Pharma Co., Astellas Pharma Inc., Ayumi Pharmaceutical Co., Bristol Myers Squibb Co., Ltd., Chugai Pharmaceutical Co., Ltd., Eisai Co., Ltd., Eli Lilly Japan K.K., GlaxoSmithKline K.K., Hisamitsu Pharmaceutical Co. Inc., Kyorin Pharmaceutical Co. Ltd., Mitsubishi Tanabe Pharma Co., Otsuka Pharmaceutical Co. Ltd., Pfizer Japan Inc., and Teijin Pharma Ltd., Atsuo Taniguchi: None declared, Hisashi Yamanaka Grant/research support from: $\mathrm{HY}$ has received research grant or speaker fee from AbbVie, Astellas, Ayumi, Behringer, Bristol-Meyers, Chugai, Daiichi-Sankyo, Eisai, Kaken, Nippon-Shinyaku, Novartis, Ono, Pfizer, Taisyo-Toyama, Takeda, Tanabe-Mitsubishi, Teijin Pharma, Torii, UCB, YLbio., Speakers bureau: HY has received research grant or speaker fee from AbbVie, Astellas, Ayumi, Behringer, Bristol-Meyers, Chugai, Daiichi-Sankyo, Eisai, Kaken, Nippon-Shinyaku, Novartis, Ono, Pfizer, Taisyo-Toyama, Takeda, Tanabe-Mitsubishi, Teijin Pharma, Torii, UCB, YLbio., masayoshi harigai Grant/research support from: AbbVie Japan GK, Ayumi Pharmaceutical Co., Bristol Myers Squibb Co., Ltd., Eisai Co., Ltd., Mitsubishi Tanabe Pharma Co., Nippon Kayaku Co., Ltd., and Teijin Pharma Ltd. MH has received speaker's fee from AbbVie Japan GK, Ayumi Pharmaceutical Co., Boehringer Ingelheim Japan, Inc., Bristol Myers Squibb Co., Ltd., Chugai Pharmaceutical Co., Ltd., Eisai Co., Ltd., Eli Lilly Japan K.K., GlaxoSmithKline K.K., Kissei Pharmaceutical Co., Ltd., Oxford Immuotec, Pfizer Japan Inc., and Teijin Pharma Ltd. $\mathrm{MH}$ is a consultant for AbbVie, Boehringer-ingelheim, Kissei Pharmaceutical Co., Ltd. and Teijin Pharma.

DOI: 10.1136/annrheumdis-2020-eular.974

\section{AB0258 CAROTID INTIMA-MEDIA THICKNESS AND SERUM BIOMARKERS IN PARAGUAYAN PATIENTS WITH RHEUMATOID ARTHRITIS}

V. Valinotti ${ }^{1},{ }_{\text {A. Paats }}{ }^{1}$, R. Acosta ${ }^{1}$, L. Roman ${ }^{1}$, I. Acosta-Colman ${ }^{1}$, M. Duarte ${ }^{2}$, O. Centurion ${ }^{2}$, T. Martinez ${ }^{3}$, P. De Abreu ${ }^{4}$, G. Avila ${ }^{1}$, S. Cabrera-Villalba ${ }^{1}$. ${ }^{1}$ Clinicas Hospital, Universidad Nacional de Asuncion, Rheumatology, Asunción, Paraguay; ${ }^{2}$ Hospital de Clinicas, Universidad Nacional de Asuncion, Cardiovascular Medicine, Asunción, Paraguay; ${ }^{3}$ Laboratorio Curie, Asuncion, Paraguay; ${ }^{4}$ Sociedad Paraguaya de Reumatología, Asunción, Paraguay

Background: The mechanism of increased cardiovascular risk in RA is not well understood and is independent of traditional CV risk factors. Intima-media thickness of the common carotid wall measured by ultrasonogram is a safe and useful biomarker of early stage atherosclerosis that correlates with coronary involvement; and it correlates with severity and duration of disease. Several studies have shown a relationship between inflammation markers, endothelial dysfunction markers, and carotid involvement. (1)

Objectives: To determine the presence of inflammation biomarkers and its relationship with subclinical atherosclerosis measured by carotid ultrasound, and with the clinical characteristics in patients with established Rheumatoid Arthritis (RA)

Methods: Descriptive, cross sectional, prospective study, in a Paraguayan cohort of patients with RA meeting ACR/EULAR2010 criteria. This study had two phases: the first one, included a standardized questionnaire according to the variables included in the Cardiovascular Risk project (PINV15-0346), from the National Sciences and Technology Council (CONACYT), and physical examination; the second one included laboratory sample collection performed by a specialized laboratory for serum biomarkers measurement for cardiovascular risk prediction (i.e endothelin, alpha-TNF, E-selectin, homocysteine, apolipoprotein, fibrinogen, and high sensitivity-CRP levels) and carotid ultrasound evaluation by a trained specialist, to evaluate subclinical atherosclerosis. Subclinical atherosclerosis was defined as carotid intima-media thickness (CIMT) $>0,9 \mathrm{~mm}$ and/or presence of carotid plaques. All patients signed informed consent. SPSS 23rd version was used for data analysis. Quantitative variables were presented as means and qualitative as frequencies. Chi square test was performed for comparisons between dichotomous variables and $t$ Student for continuous, and $p \leq 0.05$ for statistical significance.

Results: 100 patients were included, $87 \%$ were women, mean disease duration $130.9 \pm 102.64$ months, $77 \%$ were RF positive, and $84.4 \%$ were ACPA positive $43.4 \%$ had bone erosions, mean ESR-DAS28 was $3,42 \pm 1,1 ; 30 \%$ had remission criteria. $39 \%$ had extra-articular manifestations.

Elevated serum biomarkers were found: fibrinogen $>400 \mathrm{mg} / \mathrm{dL} 88.2 \%$, high sensitivity-CRP (hs-CRP) $>5 \mathrm{mg} / \mathrm{dL} 42.9 \%$, endothelin $>2 \mathrm{ng} / \mathrm{mL} 20 \%$, alphaTNF $>15,6 \mathrm{pg} / \mathrm{mL} 13.1 \%$, E-selectin $>79,2 \mathrm{ng} / \mathrm{mL} 6 \% .25 .3 \%$ had CIMT $>0,9 \mathrm{~mm}$ and mean CIMT was $0.68 \pm 0.25 \mathrm{~mm}$. $27.14 \%$ had carotid plaques. Patients with CIMT>1mm had higher frequency of family history of arterial hypertension $(p=0.006)$, greater mean disease duration $(p=0.0007)$, hip circumference $(p=0.014)$, blood pressure (SBP $p=0.038$, DBP $p=0.027)$, HAQ levels $(p=0,019)$ and hs-CRP levels $(p=0.013)$, also lower mean height $(p=0,04)$; while carotid plaques were related to higher homocysteine $(p=0.026)$ and hs-CRP levels $(p=0.024)$.

Conclusion: A considerable percentage of patients had subclinical atherosclerosis. Patients with $\mathrm{CIMT}>0,9 \mathrm{~mm}$ had a longer disease duration, higher HAQ levels, hip circumference, as well as higher BP. High levels of hs-CRP were more frequently related to the presence of subclinical atherosclerosis

References:

[1] Aday, A. targeting residual inflammatory risk: a shifting paradigm for atherosclerotic disease. Frontiers in cardiovascular medicine. 2019. 6:16. https:// www.ncbi.nlm.nih.gov/pmc/articles/PMC6403155/pdf/fcvm-06-00016.pdf

Disclosure of Interests: None declared

DOI: 10.1136/annrheumdis-2020-eular.6520

\section{AB0259 \\ THE RELATIONSHIP BETWEEN MICROVASCULAR DAMAGE AND DISEASE ACTIVITY IN PATIENT WITH RHEUMATOID ARTHRITIS - ASSESSMENT BY VIDEOCAPILAROSCOPY}

M. Parvu ${ }^{1}$, A. Cozos ${ }^{2}$, A. I. Biro ${ }^{1}$, H. V. Popoviciu ${ }^{1} .{ }^{1}$ George Emil Palade" University of Medicine, Pharmacy, Science and Technology, Emergency County Hospital Targu Mures, Rheumatology and Physiotherapy, Tg. Mures, Romania; ${ }^{2}$ Emergency County Hospital, Targu Mures, Rheumatology, Tg. Mures, Romania

Background: Rheumatoid arthritis (RA) is an autoimmune disease characterized by erosive synovitis (causing severe joint damage) and systemic damage. 